\title{
Aspectos clínicos e tomográficos dos microburacos maculares
}

\author{
Clinical and tomographic aspects of macular microholes
}

\author{
Fernando José de Novelli ${ }^{1}$ \\ Otacílio de Oliveira Maia Júnior ${ }^{2}$ \\ Mário Junqueira Nóbrega ${ }^{3}$ \\ Theodomiro Garrido Neto ${ }^{4}$ \\ Walter Yukihiko Takahashi ${ }^{5}$
}

Trabalho realizado no Hospital de Olhos Sadalla Amin Ghanem - Joinville (SC) - Brasil.

Médico especialista em Retina e Vítreo do Hospital de Olhos Sadalla Amin Ghanem - Joinville (SC) - Brasil.

Doutor, Especialista em Retina e Vítreo do Hospital São Rafael, Fundação Monte Tabor, Salvador (BA) Brasil; Professor Substituto da Faculdade de Medicina da Universidade Federal da Bahia - UFBA - Salvador (BA) - Brasil.

${ }^{3}$ Doutor, Professor do Curso de Medicina da Universidade da Região de Joinville - UNIVILLE - Joinville (SC) - Brasil.

${ }^{4}$ Doutor, Professor da Universidade do Estado do Amazonas - UEA - Manaus (AM) - Brasil.

Doutor, Médico Assistente e Chefe do Setor de Retina e Vítreo do Departamento de Oftalmologia do Hospital das Clínicas da Universidade de São Paulo - USP - São Paulo (SP) - Brasil.

Endereço para correspondência: Fernando José de Novelli. Rua Carlos Eberhardt, 321 - Joinville (SC) CEP 89218-160

E-mail: Fernando.novelli@gmail.com

Recebido para publicação em 10.06.2008

Última versão recebida em 24.11.2008

Aprovação em 04.12.2008

\begin{tabular}{l} 
RESUMO \\
\hline Objetivo: Descrever os aspectos clínicos e tomográficos do microburaco \\
macular. Métodos: Estudaram-se pacientes portadores de microburaco \\
macular de forma retrospectiva e observacional. Apurou-se história \\
clínica, medida de acuidade visual, biomicroscopia de polo posterior, \\
retinografia, angiofluoresceinografia retiniana e tomografia de coerência \\
óptica. Resultados: Examinaram-se oito olhos de sete pacientes portado- \\
res de microburaco macular. A idade variou entre 26 e 63 anos (média de \\
48,8 anos). Seis pacientes eram do sexo feminino (85,7\%). Cinco pacien- \\
tes apresentaram o microburaco macular no olho direito (62,5\%). Quanto \\
à sintomatologia, cinco indivíduos referiram diminuição da acuidade \\
visual (71,4\%), um referiu escotoma central (14,3\%) e um não apresentou \\
queixas visuais (14,3\%). A angiofluoresceinografia retiniana não mos- \\
trou alterações maculares em cinco dos olhos (71,4\%). À tomografia de \\
coerência óptica, os oito olhos apresentaram uma lesão foveal hiporrefle- \\
tiva e menor do que 100 micra, que acometia as camadas mais profundas \\
da retina neurossensorial. Conclusão: Microburaco macular é um peque- \\
no defeito lamelar presente na camada externa profunda da retina, que é \\
evidente à biomicroscopia macular como uma lesão arredondada averme- \\
lhada de tamanho diminuto, levando a pouca repercussão na função \\
visual, sem caráter progressivo. A história clínica, a acuidade visual, a \\
biomicroscopia de fundo e a tomografia de coerência óptica são os \\
principais elementos para a detecção e o estudo dos mecanismos fisiopa- \\
tológicos responsáveis pela sua origem e evolução.
\end{tabular}

Descritores: Perfurações retinianas/diagnóstico; Tomografia de coerência óptica/métodos; Angiofluoresceinografia

\section{INTRODUÇÃO}

O microburaco na mácula tem sido verificado em pacientes com perda leve da acuidade visual, escotoma central e/ou metamorfopsia. Apresenta-se como pequeno defeito (<150 micra), localizado na região foveal, de aspecto avermelhado com margens bem definidas e difícil delimitação da profundidade na estrutura retiniana, podendo acometer um ou ambos os olhos ${ }^{(1-2)}$. Essas lesões são consideradas distintas das alterações observadas no buraco macular idiopático, pois os microburacos se caracterizam pelo menor tamanho, mínima diminuição da acuidade visual e pela evolução clínica não progressiva ${ }^{(3)}$. Mais recentemente, pesquisadores, utilizando o tomógrafo de coerência óptica (OCT), demonstraram que o dano estrutural do microburaco é apenas intrarretiniano $^{(4)}$. 
A hipótese diagnóstica de microburaco macular deve ser considerada nos pacientes com queixa de baixa visual, escotoma central ou metamorfopsia, que apresentem lesões foveais suspeitas à fundoscopia. $\mathrm{O}$ diagnóstico de microburaco pode ser facilmente negligenciado numa investigação oftalmológica devido ao fato da maioria dos pacientes apresentarem apenas sintomas sutis, como um pequeno escotoma central, uma leve metamorfopsia ou pouca dificuldade na leitura. Quando o acometimento é bilateral, entretanto, os sintomas são mais evidentes, podendo até afetar a qualidade de vida dos pacientes.

O microburaco macular é uma entidade pouco diagnosticada, devido às alterações retinianas serem discretas e os achados tomográficos, peculiares. Este estudo objetiva avaliar portadores de microburaco macular por meio de sua acuidade visual, retinografia, angiofluoresceinografia e da tomografia de coerência óptica, abordando aspectos práticos dessa doença, assim como discutindo diagnósticos diferenciais e exames propedêuticos importantes.

\section{MÉTODOS}

O trabalho foi realizado através de estudo retrospectivo observacional de pacientes portadores de microburaco macular provenientes das regiões Norte, Nordeste, Sul e Sudeste do Brasil. Os exames propedêuticos utilizados foram: medida da acuidade visual sob protocolo refracional em $\log$ MAR (Equivalente Snellen), biomicroscopia de polo posterior com lente Volk 78, retinografia, angiofluoreseinografia e OCT "Stratus version 4.0 software" (Carl Zeiss Meditec). A história clínica de cada paciente foi cuidadosamente verificada, avaliando exposição solar, traumas oculares, comorbidades sistêmicas e outras doenças oftalmológicas.

Determinou-se o tempo de seguimento de cada paciente, sendo avaliadas alterações funcionais e estruturais nesse período, repetindo os exames complementares. As imagens obtidas por meio do OCT foram realizadas no programa de medida da espessura da retina (retinal thickness) e o cursor foi colocado manualmente no centro da fóvea, quando a depressão foveal era visível, ou na parte mais espessa no centro da mácula. Selecionaram-se três casos para a ilustração do estudo.

As variáveis quantitativas foram expressas em médias e desvios padrão; as qualitativas calcularam-se em frequências absolutas e relativas. Para a comparação entre variáveis do início e fim do seguimento, foi adotado o teste não-paramétrico de Wilcoxon ${ }^{(5)}$. O nível de significância utilizado para os testes foi de $5 \%$.

\section{RESULTADOS}

O resumo das características dos pacientes está demonstrado na tabela 1. Foram selecionados 7 pacientes ( 8 olhos) portadores de microburaco macular para o estudo. Nenhum dos pacientes teve história de trauma ocular, uso crônico de medicamentos, consumo de drogas injetáveis ou exposição ex-

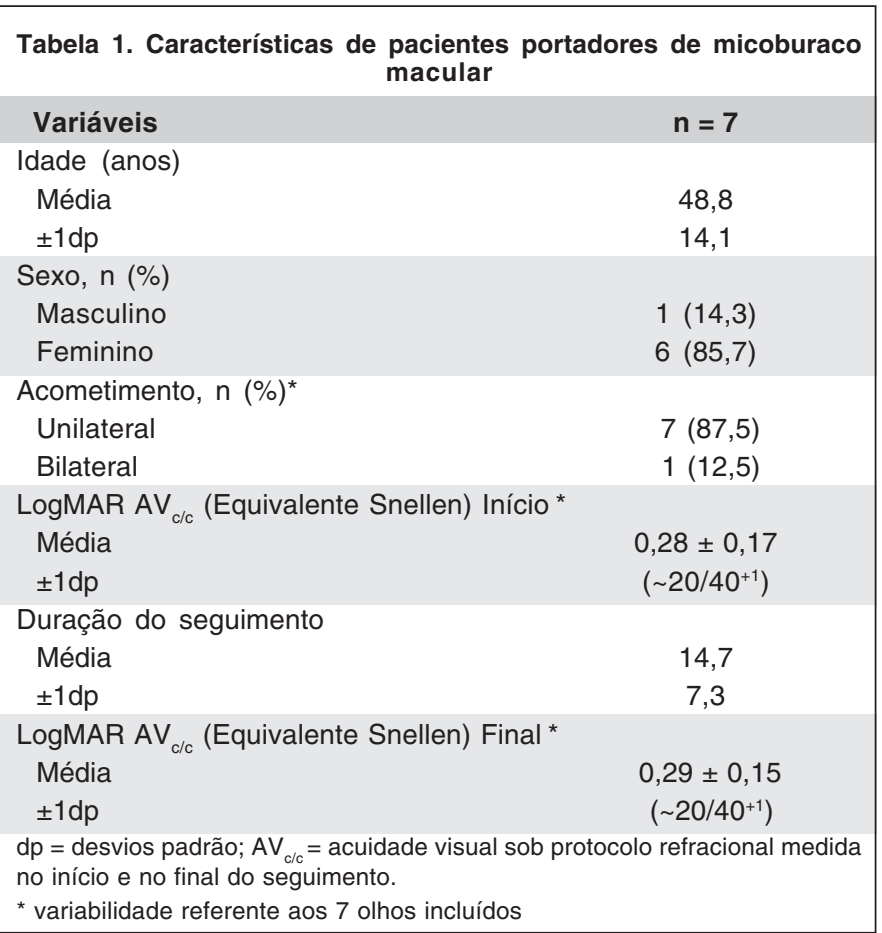

cessiva à luz solar. $\mathrm{O}$ acometimento bilateral foi em apenas um paciente, sendo que, na totalidade dos casos, o olho direito verificou-se mais afetado $(62,5 \%)$ que o esquerdo $(32,5 \%)$. A idade variou entre 26 a 63 anos, com média de 48,8 $\pm 14,1$ anos. A predominância foi do sexo feminino com seis pacientes $(85,7 \%)$. Quanto à sintomatologia, 71,4\% dos pacientes referiram diminuição da acuidade visual, 14,3\% escotoma central e 14,3\% não apresentaram queixas.

A acuidade visual em logMAR com melhor correção [média (Equivalente Snellen) $\pm d p]$, no início do seguimento, variou de 0,1 a $0,5\left[0,28\left(\sim 20 / 40^{+1}\right) \pm 0,17\right]$. Nenhum dos pacientes teve indicação de qualquer tipo de tratamento. A duração do seguimento variou de 5 a 24 meses (média de 14,7 \pm 7,3 meses), sendo determinada pela diferença entre o tempo de diagnóstico do microburaco macular e a última avaliação. No final do seguimento, a acuidade visual variou de 0,12 a $0,5\left[0,29\left(\sim 20 / 40^{+1}\right) \pm\right.$ $0,15]$, não havendo diferença estatisticamente significante da avaliação inicial $\left(P_{\text {bilateral }}=0,59\right)$ (Figuras 1 e 2$)$.

Na biomicroscopia, todos os olhos apresentaram lesão arredondada de bordos bem definidos, coloração avermelhada, profunda e de tamanho diminuto. As lesões não sofreram alterações biomicroscópicas ao longo do seguimento. Nenhum dos olhos apresentou hemorragia intrarretiniana, microaneurismas, exsudação lipídica ou buraco macular completo. Quanto às alterações angiográficas, $71,4 \%$ dos olhos não apresentaram quaisquer alterações na mácula e $28,6 \%$ apresentaram hiperfluorescência perifoveal, que não aumentava no decorrer do exame, devido a um pequeno defeito em janela do epitélio pigmentado da retina (Figura 3). Um paciente não foi submetido ao exame angiográfico por problemas alérgicos. 

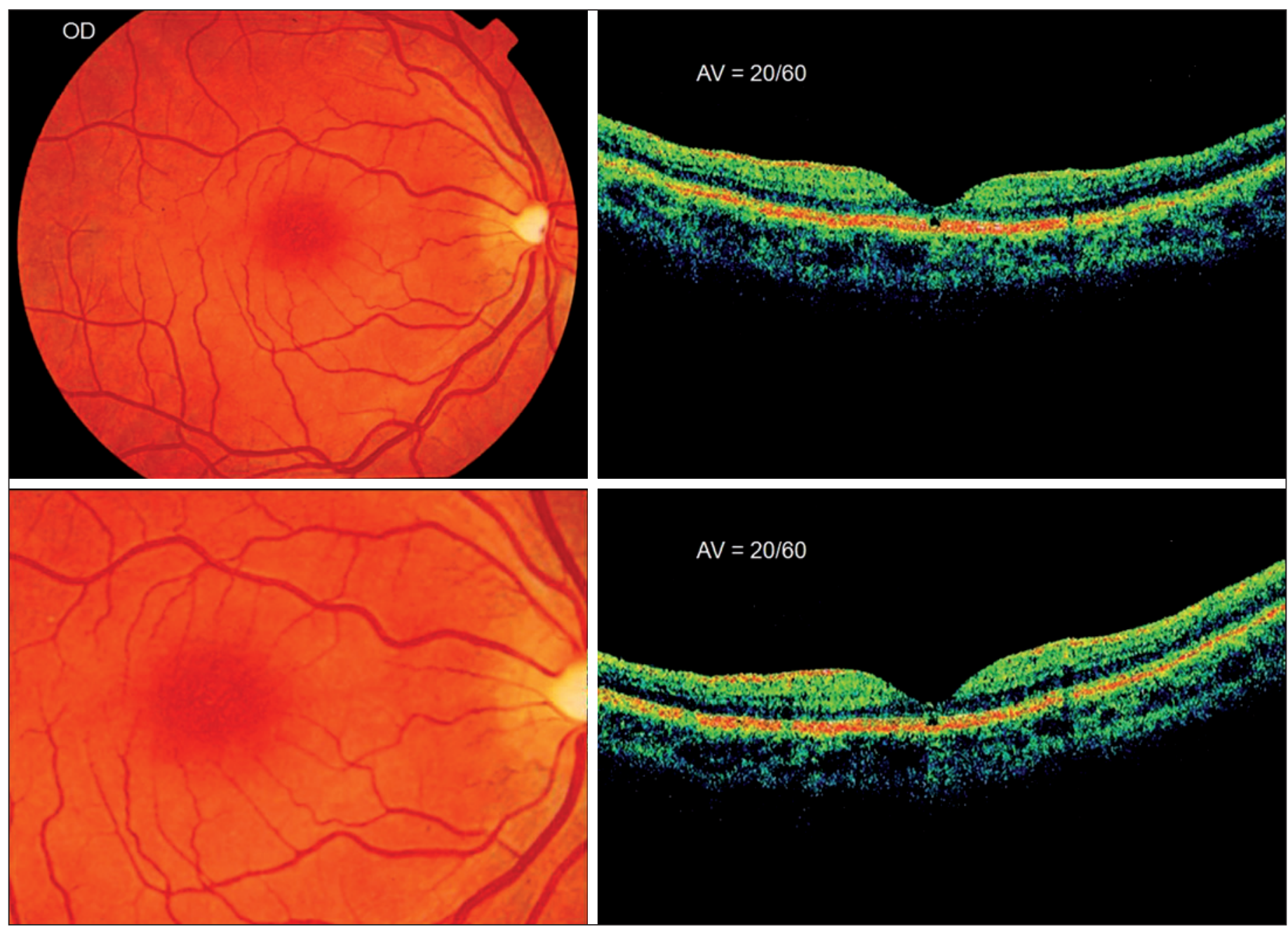

Figura 1 - Retinografia mostra alteração de coloração perifoveolar e OCT evidencia a alteração hiporreflexiva foveal profunda

Na tomografia de coerência óptica, todos os olhos apresentaram uma lesão pequena ( $<100$ micra) e hiporrefletível na região foveal, que acometia as camadas mais profundas da retina, rompendo a linha de alta refletividade externa correspondente à junção dos segmentos interno e externo dos fotorreceptores (Figuras 1, 2 e 3). Não se detectaram alterações da junção hialóide posterior - membrana limitante interna - nos pacientes examinados.

\section{DISCUSSÃO}

O microburaco macular é uma entidade patológica pouco conhecida e diagnosticada. Em geral, o paciente apresenta discreta perda de acuidade visual, escotoma central e, menos comumente, metamorfopsia. A alteração fundoscópica é tênue, porém característica, e o diagnóstico é confirmado com a tomografia de coerência óptica.

Ao exame fundoscópico todos os pacientes apresentaram lesão arredondada, de borda bem definida, coloração averme- lhada, profunda e de tamanho diminuto ( $<100$ micra) na região foveal. Esta lesão foi única e não acompanhada de alterações vasculares, hemorrágicas ou exsudativas. Por todo o período de seguimento, tanto a visão quanto a alteração fundoscópica mantiveram-se estáveis, corroborando o caráter não-progressivo desta entidade patológica. No estudo de Emerson ${ }^{(6)}$, avaliaram-se vinte e oito olhos durante (39+/- 9 meses) com visão e sintomas estáveis durante todo período de seguimento.

Em nosso estudo, a angiofluoresceinografia não revelou alteração macular em 5 olhos $(71,4 \%)$. Dois olhos apresentaram hiperfluorescência transmitida foveal, devido a um pequeno defeito em janela do epitélio pigmentar da retina. A ausência de fluorescência pode ocorrer tanto pelo defeito diminuto do epitélio pigmentar da retina quanto pela possibilidade de integridade desta camada. Em estudos prévios, a alteração angiográfica macular é pouco evidenciada ${ }^{(2,6-7)}$.

A tomografia de coerência óptica (OCT) é um exame de extrema importância para conclusão diagnóstica do microburaco macular. No presente estudo, todos os olhos apresentaram um defeito hiporreflexivo na região foveal de peque- 

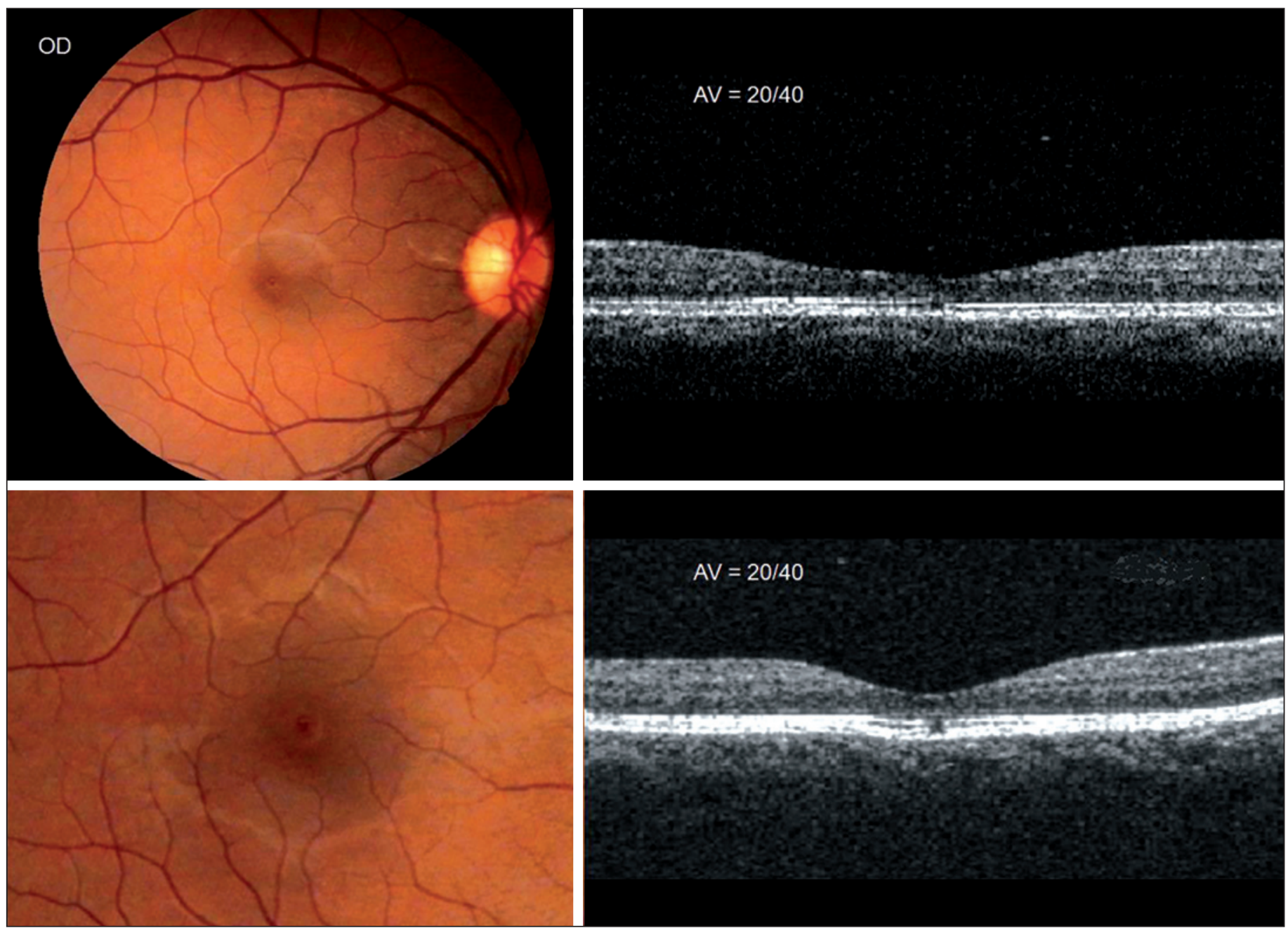

Figura 2 - Retinografia mostra nítida lesão pequena arredondada de coloração avermelhada e OCT confirma lesão foveolar hiporreflexiva em camada externa da retina

na dimensão (<100 micra), situado na camada profunda da retina, mais especificamente na linha de alta refletividade externa, que corresponde à junção dos segmentos interno e externo dos fotorreceptores, corroborando os resultados de Emerson $^{(6)}$ e Zambarakji ${ }^{(2)}$; sendo que este último avaliou dezoito olhos ao OCT com lesão similar aos casos aqui descritos.

Em nenhum caso o OCT revelou descolamento parcial do vítreo posterior ou tração anteroposterior exercida pela membrana hialóide posterior. Inclusive esta não foi identificada em nenhuma imagem tomográfica, o que impossibilitou precisar a situação anatômica do vítreo posterior.

Os principais diagnósticos diferenciais do microburaco macular são a maculopatia $\operatorname{solar}^{(8)}$, o trauma ocular direto ou indireto $^{(9)}$, e o buraco macular idiopático ${ }^{(3)}$. No entanto, nenhum dos sete pacientes avaliados neste estudo apresentou histórico de trauma ocular, exposição retiniana direta à luz solar, espontânea ou provocada por visualização de eclipse, uso de drogas ou patologias psiquiátricas.
Nos casos de diagnóstico de buraco macular idiopático de grau 1, a tomografia de coerência óptica pode mostrar alteração hiporreflexiva cística profunda, na região foveal $^{(3)}$, semelhante aos casos de microburaco macular. No entanto, diferentemente do que ocorre no microburaco macular, nos estágios iniciais do buraco macular idiopático, costuma haver nítida aderência da membrana hialóide posterior à retina, o que provoca o aplanamento da região foveal. Nos estágios mais avançados, o buraco macular idiopático apresenta defeito em toda as camadas retinianas, levando à baixa visual mais acentuada e de caráter progressivo, o que também não ocorre no microburaco macular $^{(3)}$.

Em geral, o microburaco macular não apresenta fator causal direto relacionado. Isto leva a supor que a lesão pode ser primária, devido a mecanismos fisiopatológicos não estabelecidos atualmente. No entanto é provável que a luz solar e o trauma ocular direto ou indireto sejam algumas de suas principais etiologias secundárias. Isso se pode afirmar pelo tipo 

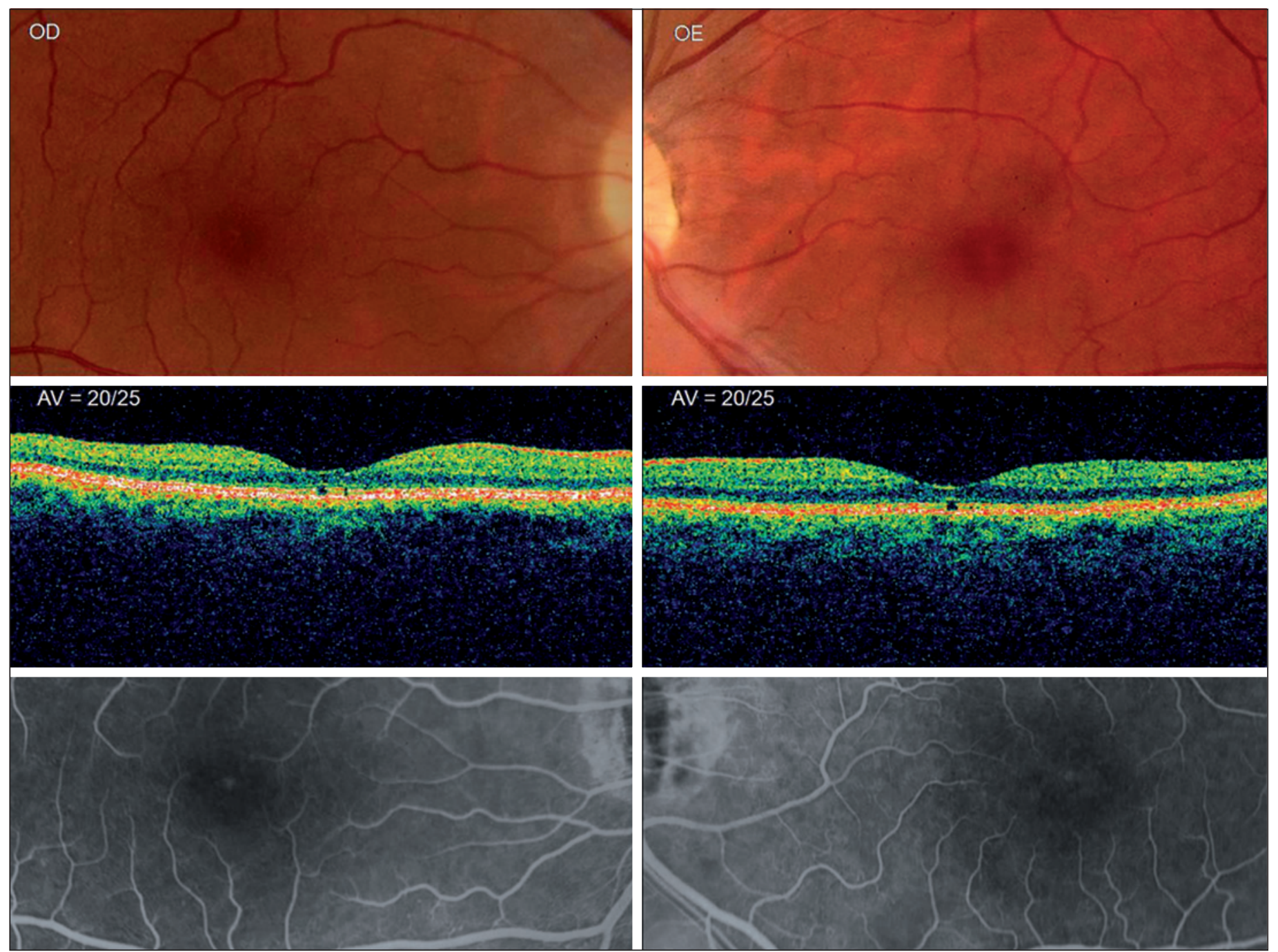

Figura 3 - Retinografia mostra discreta alteração puntiforme bem definida avermelhada, bilateral; OCT apresenta alteração hiporreflexiva profunda subfoveolar em ambos os olhos e a angiofluoresceinografia mostra defeito em janela foveal bilateral

de lesão que determinam e pelos antecedentes, muitas vezes negligenciados pelos pacientes.

Não há dúvida de que esta patologia é sub-diagnosticada, tanto pela alteração fundoscópica tênue, que necessita de exame biomicroscópico cauteloso e minucioso da região macular para a observação do defeito retiniano, quanto pela discreta repercussão na acuidade e qualidade visual. Com o exame fundoscópico detalhado e, sobretudo com a disponibilidade do tomógrafo de coerência óptica, cada vez mais teremos a possibilidade de fazer o diagnóstico e estudar os mecanismos fisiopatológicos responsáveis na gênese desta entidade patológica.

\section{ABSTRACT}

Purpose: To describe the clinical aspects and evaluate optical coherence tomography of macular microholes. Methods: Seven patients were assessed ( 8 eyes) with microholes of the macula. All patients underwent complete eye examination, fundus photography, fluorescent angiography and OCT-3 imaging. Results: Ages ranged from 26 to 69 years. Six patients were female (85.7\%) and five of them had microhole in the right eye. The presenting symptom was decrease in visual acuity (71.3\%) and central scotoma in (14.3\%). Five eyes $(71.4 \%)$ had no defects shown by fluorescent angiography. A defect in the outer retina was demonstrated in all eyes on optical coherence tomography. The lesions were nonprogressive. Conclusion: Macular microholes are small lamellar defects in the outer retina. The condition is nonprogressive, generally unilateral and compatible with good visual acuity. Fundus biomicroscopy associated with an optical coherence tomography are the main elements in the diagnosis and study of this pathology.

Keywords: Retinal perforations/methods; Tomography, optical coherence/methods; Fluorescein angiography 


\section{REFERÊNCIAS}

1. Cairns JD, McCombe MF. Microholes of the fovea centralis. Aust N Z J Ophthalmol. 1988;16(2):75-9.

2. Zambarakji HJ, Schlottmann P, Tanner V, Assi A, Gregor ZJ. Macular microholes: pathogenesis and natural history. Br J Ophthalmol. 2005;89(2): 189-93.

3. Gass JD. Idiopathic senile macular hole. Its early stages and pathogenesis Arch Ophthalmol. 1988;106(5):629-39.
4. Douglas RS, Duncan J, Brucker A, Prenner JL, Brucker AJ. Foveal spot: a report of thirteen patients. Retina. 2003;23(3):348-53.

5. Rosner B. Fundamentals of biostatistics. $2^{\text {nd }}$ ed. Boston (Mass): PWS-Kent;1986.

6. Emerson GG, Spencer GR, Klein ML. Macular microholes. Retina. 2007;27(5):595-600.

7. Reddy CV, Folk JC, Feist RM. Microholes of the macula. Arch Ophthalmol. 1996; 114(4):413-6.

8. Huang SJ, Gross NE, Costa DL, Yannuzzi LA. Optical coherence tomography findings in photic maculopathy. Retina. 2003;23(6):863-6.

9. Kelley JS, Hoover RE, George T. Whiplash maculopathy. Arch Ophthalmol. 1978;96(5):834-5

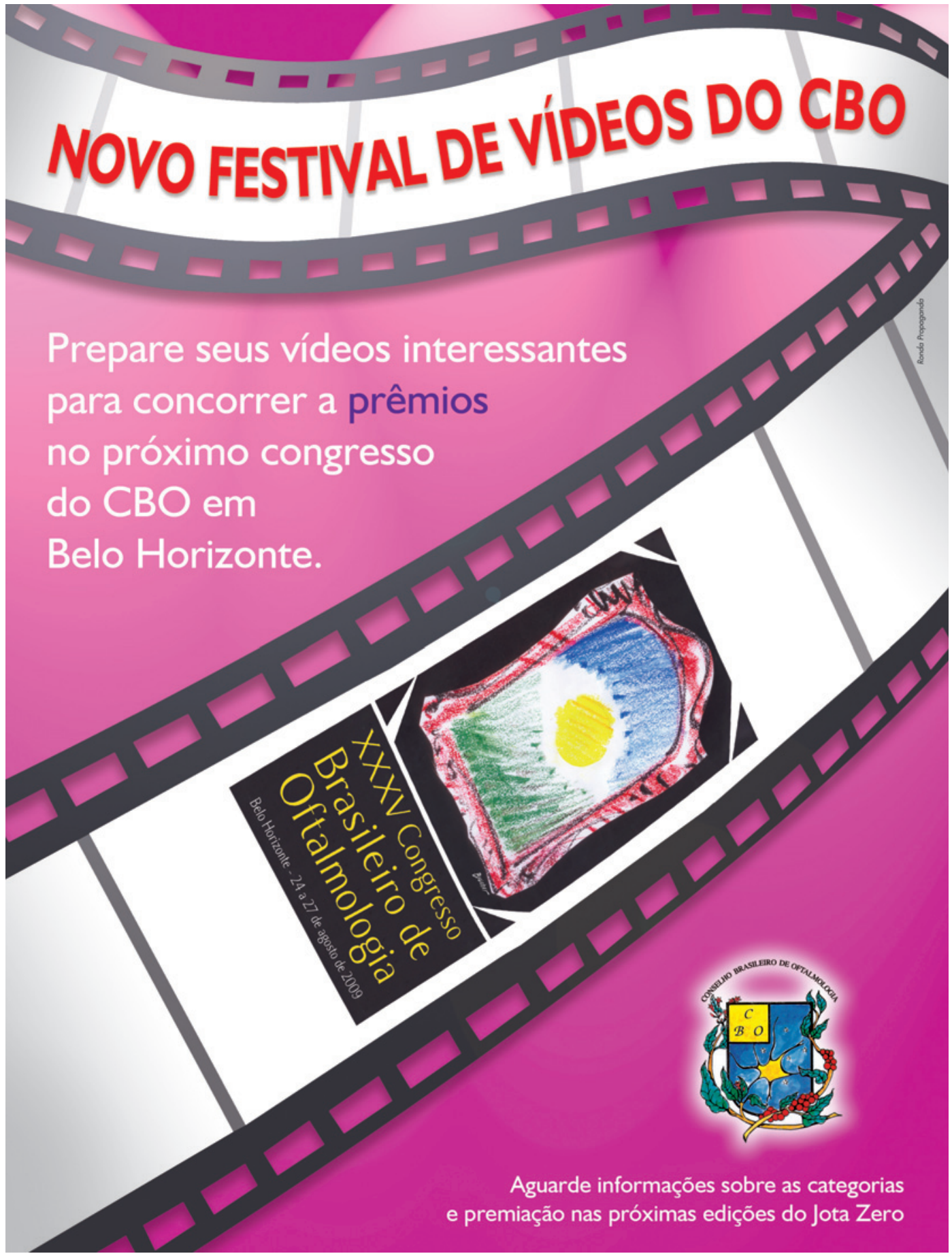

